\title{
A national curriculum in anesthesia: rationale, development, implementation, and implications
}

\author{
Salvatore M. Spadafora, MD • Patricia Houston, MD • \\ Mark Levine, MBBCh
}

Received: 10 February 2012/Accepted: 12 April 2012/Published online: 8 May 2012

(C) Canadian Anesthesiologists' Society 2012

In 2010, the Specialty Committee of the Royal College of Physicians and Surgeons of Canada (RCPSC) approved the use of the National Postgraduate Medical Education Curriculum in Anesthesia as a companion to the description of the Role of Medical Expert in the Objectives of Training Requirements (OTRs). The National Curriculum in Anesthesia represents the first of its kind in postgraduate medicine in Canada and will be a model for other national curricula moving forward. A collective consensus was reached amongst the Association of Canadian University Departments of Anesthesia (ACUDA) program directors and the RCPSC Specialty and Examination Boards that the time had come for a national curriculum. Ironically, the concept of developing a national curriculum in anesthesia was not new. The first efforts at developing a national curriculum in anesthesia began in 1971. A committee was established through the University Departments of Anesthesia (now known as ACUDA) to build a curriculum that would align curriculum objectives to specialist national exam criteria. In their commentary in 1974, Green et al. articulated the same rationale as is the impetus for the current project. Several sound reasons exist to create the standardized national curriculum. Over time, residents

S. M. Spadafora, MD ( ()

Postgraduate Medical Education, University of Toronto, 500 University Avenue, Suite 602, Toronto, ON M5G 1V7, Canada

e-mail: sal.spadafora@utoronto.ca

P. Houston, MD

St. Michael's - Li Ka Shing International Healthcare Education Centre, Toronto, ON, Canada

M. Levine, MBBCh

Department of Anesthesia, University of Toronto, Toronto, ON, Canada have found it more difficult to prepare for national examinations given the increasing overlap with other specialty disciplines in medicine and general surgery. ${ }^{1}$ Furthermore, the Examination Board did not have a well-defined curriculum around which to define the examination. With 17 different curricula driving one national exam, there was no assurance that anesthesia residents in all centres were being exposed to the specific elements of the medical expert competencies. With the growing demands of social accountability, there is increased pressure for programs to adapt to meet the challenges of aging populations, continuing change in health care systems, and complex work environments. While all postgraduate residency anesthesia programs were based on OTRs set out by the RCPSC and were all well developed prior to the National Curriculum in Anesthesia, there remained a gap in standardized curricular content to prepare residents for a standardized national exam in anesthesia. The introduction of competencies and the development of training models outside of the traditional apprenticeship approach to medical education were creating gaps for postgraduate medical educators in terms of matching objectives to assessment and learning strategies. The development of the recent National Curriculum in Anesthesia is a milestone in narrowing this gap.

\section{Background}

The history of medical teaching and learning is grounded in the apprenticeship model. While teaching and learning medicine were once described as "see one, do one, teach one", there has been a transition in recent years to a more formal process grounded in the development and maintenance of competence. ${ }^{2}$ A "competency framework" is a 
"student-centred" structure that sets out and defines how each resident can demonstrate the ability to perform a role (competency) required to be a successful and effective physician. Curriculum design in this new era of medical education " 'begins with the end in mind' and focuses on the competencies needed by graduates of medical education to meet the needs of those they serve, and effect the desired outcomes in health care." 3

Kern et al. (1998) described a curriculum as "a planned educational experience". 4 In order to develop a curriculum that will provide equally beneficial experiences for each trainee across the country, it is critical to establish an effective curriculum design. The basics of curriculum design have always incorporated course content, program aims and objectives, course learning outcomes, teaching and learning activities, and assessment methods and tools. The way an educator views the components and establishes the process of these elements dictates the curriculum design. ${ }^{5,6}$ While there has been Canadian research in the area of education in anesthesia over the last 30 years, it has been determined through initial literature searches that focused mainly on resident selection, assessment, and simulation, and there was little focus on curriculum design in anesthesia. In Christopher Eagle's article, Anaesthesia and Education, published in 1992, he summarized the state of curriculum in anesthesia by stating that "medical education must place greater emphasis on learning than on teaching; on students than on teachers; on problem solving than on fact-recall; on the community than on its hospitals; and on disease prevention than on illness".

\section{Curriculum development and implementation process}

The ACUDA Postgraduate Education Committee took on the responsibility of developing the National Curriculum in Anesthesia. The Committee was comprised of program directors from all anesthesia residency programs, chairs of exam boards, and the Chair of the Royal College Specialty Committee. ${ }^{A}$ A modified Delphi approach was used in developing the National Curriculum. The medical education literature and relevant anesthesia texts were reviewed to identify major topic areas that should be covered in the curriculum. The starting point for the National Curriculum in Anesthesia was an adaptation of the curriculum of the University of Toronto's residency program, which at the time was the only comprehensive documented form of anesthesia curriculum. The curricula of all 17 Canadian university anesthesia programs were also reviewed to

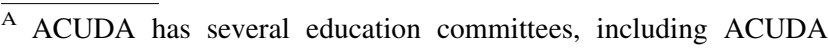
Undergraduate Education Committee and ACUDA Continuing Education and Professional Development Committee.
}

determine similarities and differences in content, specific objectives and assessment methods. In June 2006, the first template of the topic areas to shape the National Curriculum in Anesthesia was developed from this comprehensive review. Afterward, the ACUDA Education Committee developed a competency-based framework that could be used for all major medical expert roles. The identified topic areas were sent out to program directors for review and consensus, and competencies for each of the topic areas were then developed in consultation with a variety of medical educators and content experts in the field. Separate competency-based templates for "clinical" and "basic team" areas were constructed by members of the ACUDA Education Committee to provide visual assistance for aligning topic areas with required competencies. Once approved, the competencies were reviewed in accordance with the identified topic areas to ensure relevance and appropriate matching to the objectives. In 2010, the finalized curriculum was distributed to all programs, trainees, the RCPSC Specialty Committee in Anesthesia, and the Royal College Examination Board for approval. The project team performs regular systematic reviews of the current National Curriculum in Anesthesia to ensure that objectives and competencies are current and relevant and keeping in line with the national examination. ${ }^{\mathrm{B}}$

While the project took approximately three years to complete, a primary goal of the initiative was to ensure that the finalized National Curriculum in Anesthesia is centred on learners and comprises competencies and objectives that reflect the mission and rationale behind the topic areas. The establishment of the specific competencies and objectives is an important step as it acts as a guide to the development of effective learning outcomes, assessment tools, and methods. Furthermore, the selection of educational learning strategies should be harmonious with the learning objectives. When designing any medical education curriculum, it is critical that medical educators be cognizant that learners come with many different learning styles, thus increasing the need to utilize more than one educational learning method. The learner's experience benefits from exposure to multiple educational learning methods, but curriculum designers must also make certain that proper resources and materials are in place to support and ensure the smooth implementation of each educational learning strategy. Each institution across Canada will have varying access to resources and materials to support learning strategies; for that reason, the design of the National Curriculum in Anesthesia is purposeful in not prescribing the teaching methodology.

\footnotetext{
B Levine M, Murphy P, Fox J, Wong C. Development of a Medical Expert National Curriculum in Anesthesiology. Poster session presented at International Conference on Residency Education (ICRE); 2011 Oct 22-25; Quebec City, QC, Canada.
} 
A challenge that reportedly arises when any new change in postgraduate medicine curriculum is implemented is not having support from the respective specialty societies or governing bodies. ${ }^{8}$ With 17 different curricula, support and collaboration were needed during the implementation of the National Curriculum in Anesthesia as there was high potential for slight variations in language and in the explanation of certain medical expert components. In addition to external support, it is also critical to have internal support from faculty and administration with the implementation of any new curriculum. Along with support, curriculum designers must consider and monitor change management and the manner in which the implementation of a new curriculum affects staff and faculty. Incorporating faculty development strategies is a way to minimize challenges with implementation as it is the faculty who will teach and embrace the new curriculum. Faculty must be educated and informed around the purpose, objectives, and rationale of a new curriculum. These components are critical to successful implementation. ${ }^{9}$

\section{Future work}

The medical education system is not static; it is always changing and therefore requires consistent review and feedback. The way in which programs and specialties receive feedback from the licensing board in Canadian postgraduate medicine requires increased partnership. Given the infancy of the National Curriculum in Anesthesia, we hope to make substantive and sustained improvement in this area. Narrowing the feedback gap between the objectives of the National Curriculum in Anesthesia and national exam outcomes will be possible only when we acquire aggregated data that detail areas where residents predominantly display weakness. Identifying areas of weakness is the first step in turning them around to areas of strength.

Furthermore, while the current National Curriculum in Anesthesia has the basic foundations of the CanMEDS Medical Expert Role in place, more work and development is needed around the six non-medical expert CanMEDS Roles, i.e., Communicator, Collaborator, Manager, Scholar, Professional, and Health Advocate. In keeping with the existing National Curriculum in Anesthesia, continued curriculum development must be non-generic and tailored to competencies specific to anesthesia.

\section{Conclusion}

Almost 40 years ago, Green et al. (1974) hoped their publication would "lead individuals and departments to discuss ..., consider the desirability and practicability [of a national curriculum]" and would be an "attempt to define a National Curriculum" (p. 351). ${ }^{1}$ Their hope has now become a reality.

In summary, with the support and assistance of the RCPSC Specialty Committee, the National Curriculum in Anesthesia was completed, reviewed, and accepted by the RCPSC in 2010. The final document is currently undergoing a format review by the Office of Education. While the National Curriculum in Anesthesia acts as a curricular guide for programs at all levels and sites around the country and the Examination Board can use it to guide the national exam, it ultimately provides residents, program directors, and medical educators with a set of competencies that are necessary and critical to becoming a competent anesthesiologist.

\section{Un programme national d'études en anesthésie: justification, élaboration, mise en ouvre et implications}

En 2010, le comité des spécialités du Collège royal des médecins et chirurgiens du Canada (CRMCC) a approuvé l'utilisation du Programme national de formation médicale postdoctorale en anesthésie comme aide à la description du rôle d'Expert médical dans les exigences d'objectifs de formation. Le Programme national d'études en anesthésie représente le premier de son genre en médecine postdoctorale au Canada et dans l'avenir servira de modèle aux autres programmes nationaux. Un consensus a été atteint entre les directeurs de programmes de l'association canadienne universitaire des départements d'anesthésie (ACUDA), le Bureau des examinateurs et les comités des spécialités du CRMCC pour déterminer qu'il était temps d'avoir un programme national d'études. Ironiquement, le concept d'élaboration d'un programme national d'études en anesthésie n'est pas neuf. Les premières tentatives d'élaboration d'un programme national d'études en anesthésie remontent à 1971. Un comité fut créé par les départements universitaires d'anesthésie (maintenant connu sous l'acronyme d'ACUDA) pour construire un programme d'études qui s'alignerait sur les objectifs du programme sur les critères de l'examen national de spécialité. Green et coll. formulaient en 1974 la même justification que celle qui est à la base du regain d'intérêt pour le projet actuel. Il existe quelques bonnes raisons pour créer un programme d'études national standardisé. Au fil des années, les résidents ont éprouvé davantage de difficultés à se préparer pour les examens nationaux, en raison d'un chevauchement croissant avec d'autres 
disciplines de spécialités médicales et de chirurgie générale. ${ }^{1}$ De plus, le Bureau des examinateurs ne disposait pas d'un programme clairement défini autour duquel établir l'examen. Avec 17 programmes d'études différents menant au même examen national, il n'y avait aucune garantie que les résidents en anesthésie de tous les centres aient été exposés aux éléments spécifiques de compétences pour le rôle d'expert médical. Avec les demandes croissantes de responsabilité sociale, il y a une pression de plus en plus forte sur les départements pour qu'ils s'adaptent aux défis posés par des populations vieillissantes, par des systèmes de soins de santé continuellement changeants et par des environnements professionnels complexes. Bien que tous les programmes de résidence postdoctorale en anesthésie aient été basés sur des exigences d'objectifs de formation définis par le CRMCC et qu'ils aient été bien développés avant l'élaboration du programme national d'études en anesthésie, il persistait un vide dans le contenu du programme standardisé pour la préparation des résidents à un examen national standardisé en anesthésie. L'introduction des compétences et l'élaboration de modèles de formation en dehors des approches traditionnelles d'apprentissage en matière d'éducation médicale ont créé des vides pour les enseignants en médecine postdoctorale quand il s'est agi de faire correspondre les objectifs à des stratégies d'apprentissage et d'évaluation. L'élaboration du récent programme national d'études en anesthésie est un jalon destiné à combler ce vide.

\section{Contexte}

L'histoire de l'enseignement et de l'étude de la médecine est ancrée dans le modèle d'apprentissage. Alors que l'enseignement et l'étude de la médecine ont été autrefois décrits par «le voir, le faire, l'enseigner », il y a eu ces dernières années une transition vers des processus plus formels s'enracinant dans le développement et le maintien de compétences. ${ }^{2}$ Un « cadre de compétences » est une structure «centrée sur l'étudiant»qui établit et définit comment chaque résident peut démontrer sa capacité à remplir un rôle (compétence) requis pour être un médecin efficace. La conception d'un programme d'études dans cette nouvelle ère de l'éducation médicale " "commence avec la finalité en vue" et se focalise sur les compétences que les diplômés de l'enseignement médical devront avoir pour répondre aux besoins de ceux qu'ils servent et produisent les résultats souhaités dans les soins de santé ». ${ }^{3}$

Kern et coll. (1998) ont décrit le programme d'études comme étant une « expérience éducative programmée ». ${ }^{4}$ Afin d'élaborer un programme d'études qui procurera des expériences également bénéfiques pour chaque stagiaire à travers tout le pays, il est essentiel de concevoir un schéma efficace de programme d'études. Les principes de base de la conception d'un programme d'études ont toujours incorporé le contenu des cours, les buts et objectifs du programme, la finalité des apprentissages, les activités d'enseignement et d'étude, ainsi que les méthodes et outils d'évaluation. La façon dont un éducateur voit les composants et détermine le traitement de ces éléments dicte la conception du programme. ${ }^{5,6}$ Bien qu'il y ait eu de la recherche au Canada dans le domaine de la formation en anesthésie au cours des 30 dernières années, elle a été dominée surtout au départ par des revues de la littérature centrées sur la sélection des résidents, l'évaluation et la simulation, et il y a eu peu d'intérêt pour la conception du programme en anesthésie. Dans son article Anesthésie et éducation, publié en 1992, Christopher Eagle a résumé l'état du programme d'études en anesthésie en déclarant que «l'éducation médicale doit insister davantage sur l'étude que sur l'enseignement, sur les étudiants que sur les enseignants, sur la résolution des problèmes que sur le rappel des faits, sur la collectivité que sur ses hôpitaux, et sur la prévention des maladies plutôt que sur la maladie ».?

\section{Élaboration d'un programme d'études et processus de mise en ouvre}

Le Comité d'éducation postdoctorale de l'ACUDA a pris en charge l'élaboration du Programme national d'études en anesthésie. Le Comité était constitué des directeurs de programmes de tous les programmes de résidence en anesthésie, des présidents des jurys d'examens et du président du Comité des spécialités du Collège Royal. ${ }^{\mathrm{A}}$ Une approche de Delphi modifiée a été utilisée pour l'élaboration du programme national d'études. La littérature sur l'éducation médicale et les textes pertinents pour l'anesthésie ont été examinés afin d'identifier les principaux domaines qui devaient être couverts par le programme. Le point de départ du programme national d'études en anesthésie a été une adaptation du programme de résidence de l'Université de Toronto qui, à ce moment-là, était le seul programme d'anesthésie documenté de façon complète. Les programmes d'anesthésie des 17 universités canadiennes ont également été passés en revue pour identifier les similitudes et les différences en termes de contenu, objectifs spécifiques et méthodes d'évaluation. En juin 2006, la première ébauche des domaines à couvrir pour donner forme au Programme national d'études en

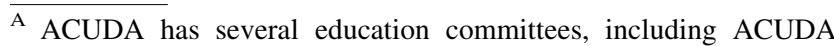
Undergraduate Education Committee and ACUDA Continuing Education and Professional Development Committee.
} 
anesthésie prenait corps à partir de cette analyse approfondie. Ensuite, le comité d'éducation de l'ACUDA a élaboré un cadre basé sur les compétences qui pourrait être utilisé pour toutes les principales fonctions d'expert médical. Les domaines identifiés ont été envoyés aux directeurs de programmes pour analyse et consensus, et les compétences nécessaires pour chaque domaine ont alors été développées en collaboration avec de nombreux enseignants en médecine et des experts de contenus dans ce domaine. Des matrices distinctes basées sur les compétences pour les domaines « cliniques» et d' « équipe de base » ont été constituées par les membres du comité d'éducation de l'ACUDA afin de contribuer à aider, sous une forme visuelle, la mise en correspondance des domaines et des compétences nécessaires. Une fois approuvées, les compétences ont été revues conformément aux domaines identifiés pour s'assurer qu'elles étaient pertinentes et correspondaient de façon appropriée aux objectifs. En 2010, le programme finalisé a été distribué à tous les départements universitaires, aux stagiaires, au comité des spécialités en anesthésie du CRMCC et au jury d'examen du Collège royal pour approbation. L'équipe du projet procède à des révisions systématiques du Programme national d'études actuel en anesthésie pour s'assurer que les objectifs et compétences restent actuels et pour rester adapté à l'examen national. ${ }^{\mathrm{B}}$

Bien qu'il ait fallu environ trois ans pour mener à bien ce projet, l'un des objectifs principaux de l'initiative était de s'assurer que le Programme national finalisé d'études en anesthésie était centré sur les étudiants et incluait les compétences et objectifs reflétant la mission et la justification sous-tendant les domaines d'intérêt. L'établissement de compétences et objectifs spécifiques est une étape importante qui sert de guide à l'élaboration de la finalité des apprentissages, d'outils d'évaluation et de méthodes efficaces. En outre, le choix des stratégies éducatives d'apprentissage doit se faire en harmonie avec les objectifs d'apprentissage. Lors de la conception de tout programme d'études médicales, il est essentiel que les enseignants soient conscients du fait que les apprenants arrivent avec des styles d'apprentissage très différents, ce qui renforce le besoin d'utiliser plus d'une seule méthode éducative. L'expérience de l'apprenant s'enrichit par une exposition à de multiples méthodes éducative d'apprentissage, mais les concepteurs du programme doivent aussi s'assurer qu'ils disposent de ressources et de matériel appropriés pour appuyer et assurer une mise en œuvre en douceur de chaque stratégie éducative d'apprentissage. Chaque

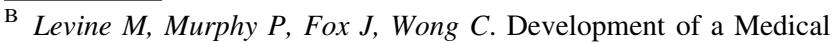
Expert National Curriculum in Anesthesiology. Poster session presented at International Conference on Residency Education (ICRE); 2011 Oct 22-25; Quebec City, QC, Canada.
}

institution canadienne aura un accès variable aux ressources et au matériel nécessaires pour soutenir les stratégies d'apprentissage; pour cette raison, le plan du Programme national d'études en anesthésie veille à ne pas imposer de méthodologie d'enseignement.

Un défi qui fait surface, semble-t-il, lors de la mise en œuvre de chaque nouveau changement au programme d'études postdoctoral est de ne pas disposer du soutien des sociétés savantes ou des organes directeurs des spécialités concernées. $^{8}$ Avec 17 programmes différents, il est nécessaire de disposer d'un soutien et de collaboration lors de la mise en œuvre du Programme national d'études en anesthésie car il y a un grand risque d'être confronté à de légères variantes dans le langage utilisé et l'explication de certains composants du rôle d'Expert médical. En plus d'un soutien extérieur, il est également essentiel de disposer d'un soutien interne de la part du corps professoral et de l'administration pour la mise en œuvre de tout nouveau programme. Tout en donnant ce soutien, les concepteurs du programme doivent envisager et surveiller la gestion des changements, ainsi que la façon dont la mise en place du nouveau programme affecte le personnel et le corps professoral. Inclure les stratégies de développement du corps professoral est un moyen de minimiser les défis de la mise en œuvre, car c'est ce corps professoral qui enseignera et adoptera le nouveau programme. Le corps professoral doit être formé et informé du but, des objectifs et de la justification du nouveau programme. Ces éléments sont essentiels à une mise en œuvre réussie. ${ }^{9}$

\section{Travail futur}

Le système d'éducation médicale n'est pas statique; il est toujours en mouvement et doit donc être constamment revu et recevoir un retour d'informations. La façon par laquelle les programmes et les spécialités reçoivent un retour d'information de la part de l'organisme d'attribution du droit d'exercice en médecine postdoctorale au Canada nécessite un plus grand partenariat. Compte tenu du très jeune âge du Programme national d'études en anesthésie, nous espérons pouvoir faire des progrès substantiels et continus dans ce domaine. Il ne sera possible de resserrer l'écart de feedback entre les objectifs du Programme national d'études en anesthésie et les résultats de l'examen national que lorsque nous aurons acquis des données agrégées détaillant les domaines dans lesquels les résidents manifestent principalement des faiblesses. Identifier les zones de faiblesse est la première étape pour en faire des zones de force.

De plus, alors que l'essentiel du rôle d'expert médical CanMEDS se retrouve actuellement dans le Programme national d'études en anesthésie, on doit continuer à 
travailler et à développer les six autres rôles CanMEDS, à savoir, Communicateur, Collaborateur, Gestionnaire, Érudit, Professionnel et Promoteur de la santé. Tout en conservant le Programme national d'études en anesthésie existant, un développement continu du programme doit être non générique et taillé sur mesure pour les compétences propres à l'anesthésie.

\section{Conclusion}

Il y a presque 40 ans, Green et coll. (1974) espéraient que leur article «conduirait les individus et les départements à débattre..., envisager le caractère souhaitable et faisable (d'un programme national) » et qu'il y aurait une « tentative de définir un Programme d'études national » (p. 351). ${ }^{1}$ Leur espoir est aujourd'hui devenu réalité.

En résumé, avec le soutien et l'aide du Comité des spécialités du CRMCC, le Programme national d'études en anesthésie a été terminé, révisé et accepté par le CRMCC en 2010. Le document définitif est actuellement en cours de révision par le Bureau de l'éducation. Alors que le Programme national d'études en anesthésie agit comme un guide pour les départements universitaires à tous les niveaux et partout au pays et que la Commission des examens peut l'utiliser comme guide pour l'examen national, il fournit finalement aux résidents, directeurs de programme et éducateurs en médecine un ensemble de compétences qu'il est nécessaire et essentiel d'acquérir pour devenir un anesthésiologiste compétent.

Acknowledgements The authors sincerely thank Melissa Kennedy Hynes for her contributions to this article. The authors also thank the following program directors for their contributions to the curriculum initiation and development: Fred Baxter, Rob Brown, Johanne Carrier, Francois Girard, Carolyn Goyer, Melanie Jaeger, Romana Kearney, Mark Levine, Jean-Pierre Morin, Patti Murphy, Jeremy Pridham, Mateen Raazi, Bruce Ramsey, Sal Spadafora, Joanne Todesco, Narendra Vakharia, Clinton Wong, and Linda Wynne.

Competing interests None declared.

\section{References}

1. Green CD, Otton P, Cockings EC, Dery R, Jenkins LC, Matthews $R L$. A proposed national curriculum in anaesthesia. Can Anaesth Soc J 1974; 21: 350-65.

2. Cooke M, Irby DM, Sullivan W, Ludmerer KM. American medical education 100 years after the Flexner report. N Engl J Med 2006; 355: 1339-44.

3. Frank JR, Danoff D. The CanMEDS initiative: implementing an outcomes-based framework of physician competencies. Med Teach 2007; 29: 642-7.

4. Kern DE. Curriculum development for medical education: a six step approach. Baltimore, MD: Johns Hopkins University Press; 1998 .

5. Prideaux D. ABC of learning and teaching in medicine. Curriculum design. BMJ 2003; 326: 268-70.

6. Harden RM. AMEE Guide No. 21: Curriculum mapping: a tool for transparent and authentic teaching and learning. Med Teach 2001; 23: 123-37.

7. Eagle C. Anaesthesia and education. Can J Anaesth 1992; 39: 158-65.

8. Scheele F, Teunissen P, van Luijk S, et al. Introducing competency-based postgraduate medical education in the Netherlands. Med Teach 2008; 30: 248-53.

9. Dath D, Iobst W, International CBME Collaborators. The importance of faculty development in the transition to competency-based medical education. Med Teach 2010; 32: 683-6. 